\title{
October 2014 Pulmonary Case of the Month: A Big Clot
}

\author{
G. Zacharia Reagle DO \\ Eyad Almasri MD \\ Stuart J. Maxwell MD \\ Departments of Internal Medicine, Division of \\ Pulmonary and Critical Care \\ and Emergency Medicine \\ UCSF Fresno \\ Fresno, CA
}

\section{History of Present IIIness:}

A 63 year-old man was brought to the emergency department (ED) with a report of acute onset of dyspnea. The dyspnea had started at rest less than one hour prior to ED presentation. It quickly progressed to severe respiratory distress. His initial vital signs were recorded as a BP of $124 / 69$, pulse of 112 , respiratory rate of 26 with an oxygen saturation $\left(\mathrm{SpO}_{2}\right)$ of $88 \%$ on 15 liters per minute (lpm) of oxygen via a non-rebreather mask. He was placed on non-invasive ventilation with intermittent episodes of brief desaturation into the $60 \%$ range. He was subsequently intubated without incident. Immediately following intubation he experienced a pulseless electrical activity (PEA) cardiopulmonary arrest.

\section{Past Medical History:}

- Diabetes mellitus

- Coronary Artery Disease

- Hypertension

- Deep Venous Thrombosis

\section{Past Surgical History:}

- Coronary Artery Bypass Graft - 2 vessel

\section{Medications:}

- Atorvastatin 40mg PO daily

- Insulin glargine 10u SQ daily

- Lisinopril 10mg PO daily

- Warfarin - had been stopped due to difficulty with compliance.

\section{Social History:}

- Married

- Owns and manages a series of used car lots.

- Lifetime non-smoker 
- Reports a remote history of chronic alcohol use, but quit in 2005 when he was diagnosed with coronary artery disease.

- He denied illicit drug use.

\section{Physical Exam:}

General: Intubated, on a fentanyl infusion at $50 \mathrm{mcg}$ per hour.

Vitals: BP: 122/63 HR: 123 RR: 24 T: 35.6

HEENT: NC/AT, PERRL, neck supple without JVD noted.

Lungs: equal chest expansion was noted with clear lung sounds.

Heart: Tachycardic and regular with a good S1 and S2, no murmurs or gallops were appreciated.

Abdomen: soft, obese, good bowel sounds.

Extremities: cold to the touch with no edema, or clubbing.

Neurological: Nonfocal exam with suppressed Glasgow Coma Scale after sedation for intubation.

Skin: No rashes noted.

\section{Laboratory:}

Complete Blood Count (CBC): White blood cell count (WBC) $8.8 \times 1000$ cells $/ \mu \mathrm{L}$, hemoglobin $14.6 \mathrm{~g} / \mathrm{dL}$, hematocrit 43.7 , platelets 191,000 cells $/ \mu \mathrm{L}$

Chemistry: Sodium $139 \mathrm{meq} / \mathrm{L}$, potassium $3.6 \mathrm{meq} / \mathrm{L}$, chloride $106 \mathrm{meq} / \mathrm{L}$ bicarbonate (CO2) $19 \mathrm{meq} / \mathrm{L}$, blood urea nitrogen (BUN) $20 \mathrm{mg} / \mathrm{dL}$, creatinine $0.7 \mathrm{mg} / \mathrm{dL}$, glucose $459 \mathrm{mg} / \mathrm{dL}$, magnesium $2.0 \mathrm{meq} / \mathrm{L}$, phosphorus $3.4 \mathrm{mg} / \mathrm{dL}$

Liver Function Tests: Albumin $4.1 \mathrm{~g} / \mathrm{dL}$, ALP 59 U/L, AST 30 U/L ALT 31 $\mathrm{U} / \mathrm{L}$. total bilirubin $0.4 \mathrm{mg} / \mathrm{dL}$

Coagulation: Prothrombin time 13.9 seconds, INR 1.1, activated partial thromboplastin time (aPTT) 24.2 seconds

Troponin: $0.007 \mathrm{ng} / \mathrm{ml}$

Brain naturetic peptide (BNP): $39 \mathrm{pg} / \mathrm{ml}$

Arterial Blood Gases (ABG): pH 7.27, pCO2 38, pO2 38

\section{Imaging:}

The patient was immediately taken for a chest CT pulmonary angiogram. As he was on the CT scan table, the CT technician discovered that his IV line was malfunctioning. Before the line could be replaced, he had several non-contrast chest CT cuts obtained (Figure 1). 

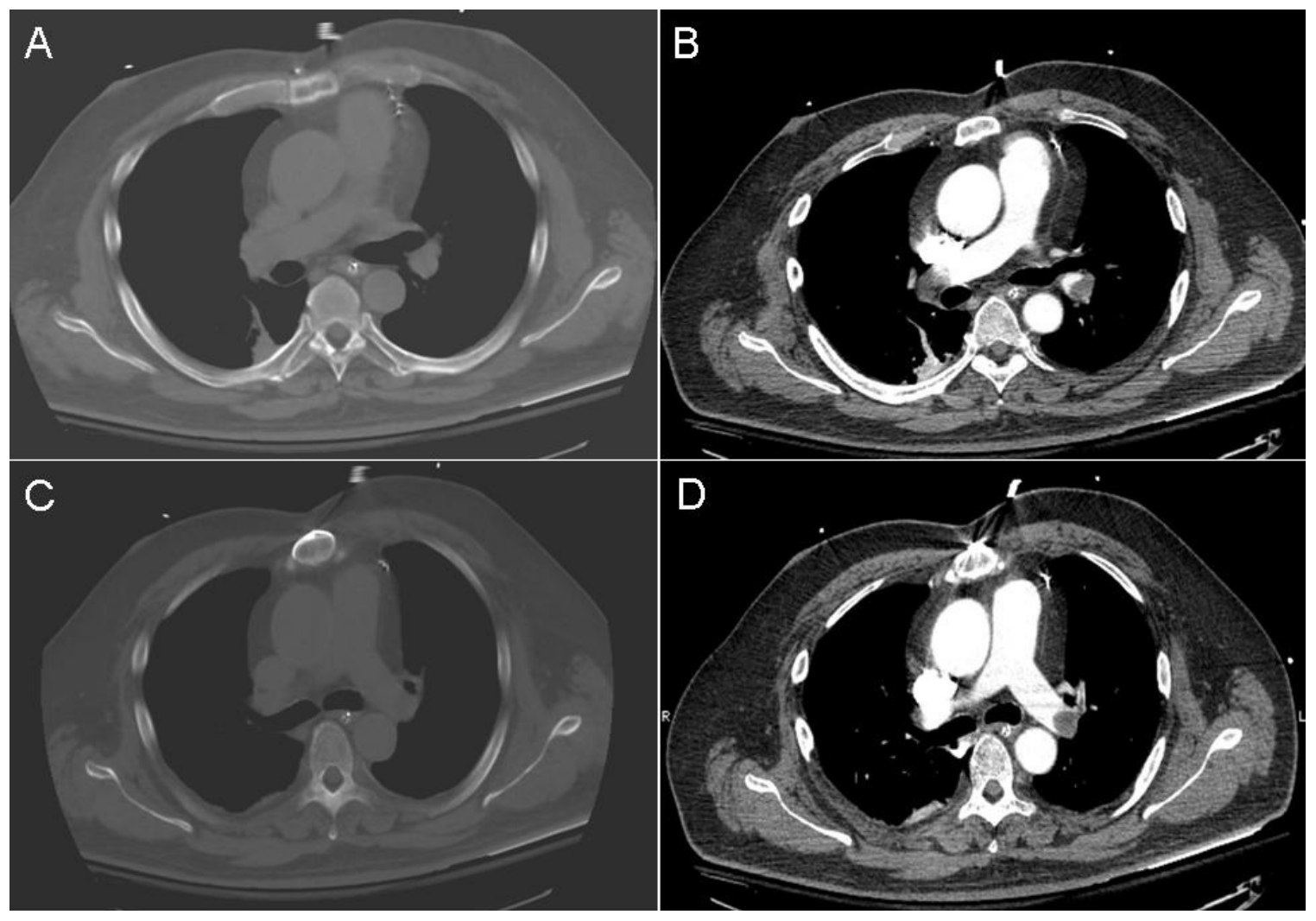

Figure 1. Images $A \& C$ are non-contrast cuts while images $B$ \& $D$ are comparison cuts that became available after the contrast study was obtained.

How often are intravascular filling defects seen on non-contrast chest CT images and what is the positive predictive value (PPV) of non-contrast images for pulmonary embolism (PE)?

1. Filling defects are often seen on non-contrast CT images and are diagnostic for pulmonary embolism.

2. Filling defects can be seen on non-contrast images but have a low PPV.

3. Filling defects indicating pulmonary embolism are never seen on noncontrast images.

4. Filling defects indicating pulmonary embolism are sometimes seen on non-contrast images and have a high PPV for PE. 


\section{Correct! \\ 4. Filling defects indicating pulmonary embolism are sometimes seen on non-contrast images and have a high PPV for PE.}

Tatco and Piedad (1) preformed a retrospective review of contrast CT images done under a PE protocol for 121 patients suspected of having pulmonary embolism. They then reviewed non-contrast images from the 25 patients who had a positive PE seen on the contrast CT images. They found that in nine of those patients an intravascular filling defect was visible even on the non-contrast images. They called this the "hyperdense lumen sign" and found the positive predictive value was $90 \%$. The "hyperdense lumen sign" was more often seen on central rather than peripheral intravascular filling defects.

In a patient with acute, massive pulmonary embolism experiencing hemodynamic instability in the absence of a clear contraindication, the treatment of choice is:

1. Emergent surgical embolectomy.

2. Fibrinolysis with systemic tissue plasminogen activator (tPA) administered through a peripheral intravenous catheter.

3. Fibrinolysis with systemic tPA administered through a pulmonary-artery catheter.

4. Therapeutic anticoagulation with intravenous heparin via a weight-based protocol. 


\section{Correct! \\ 2. Fibronolysis with systemic tPA administered through a peripheral intravenous catheter.}

The Antithrombotic Therapy and Prevention of Thrombosis Evidence Based Practice Guidelines published by the American College of Chest Physicians recommend systemic over local tPA therapy and short infusion times, (two hours) over prolonged infusion time ( $>12$ hours) (2). The use of tPA through a pulmonary-artery catheter has resulted in more bleeding at the catheter insertion site without an accelerated clot lysis. The shorter tPA infusion times have been associated with more rapid clot lysis than the longer infusion times, and longer infusion times increase the risk for bleeding.

As you are waiting for a formal CT pulmonary angiography with contrast and given the patient's clinical presentation with acute refractory hypoxemia leading to cardiopulmonary arrest, which of the following is the next step?

1. Considering his successful cardiac arrest resuscitation, initiate hypothermia protocol.

2. Initiate anticoagulation while performing review of any possible contraindications to tPA therapy.

3. Request stat interventional cardiology consultation for possible $\mathrm{PCl}$.

4. Stat cardiothoracic surgery consultation for possible embolectomy. 


\section{Correct! \\ 2. Initiate anticoagulation while performing review of any possible contraindications to tPA therapy.}

The Antithrombotic Therapy and Prevention of Thrombosis Evidence Based Practice Guidelines recommend that patients with a high clinical suspicion of acute pulmonary embolism be treated with anticoagulants as compared to withholding treatment while waiting for diagnostic test results to become available (2). Those guidelines further recommend that in patients with hypotension (defined as a systolic blood pressure $<90$ ) should also be treated with TPA if there are no contraindications. In addition, they recommend that patients at high risk for developing hypotension should be treated with tPA. However, a recent randomized, controlled trial did not demonstrate any benefit to treating patients with intermediate-risk PE with IPA and demonstrated that they are at increased risk for bleeding complications (3). The authors recommended not to use tPA unless the patient actually develops hemodynamic instability. If contraindications to tPA are present or there is a high risk of bleeding, then alternative therapies would need to be considered.

The ACCP Evidence Based Guidelines recommend catheter directed or surgical clot removal only when systemic tPA is contraindicated or has failed and the patient remains in shock. Additionally, local resources and expertise in either catheter directed or surgical clot removal needs to be available for those approaches to be considered.

\section{Case Continued:}

The patient suffered recurrent PEA cardiopulmonary arrests immediately following his return from the CT scanner. The CT images revealed large bilateral pulmonary emboli in the main pulmonary arteries. He experienced a return of spontaneous circulation following two subsequent PEA arrests with rapid and deep chest compression, hyperoxygenation and the administration of intravenous epinephrine. After he was resuscitated, he received IV tPA.

He was admitted to the Medical Intensive Care Unit (MICU) with metabolic acidosis due to diabetic ketoacidosis (DKA). This was successfully treated with intravenous fluids, insulin and electrolyte replacement. After the two hour tPA infusion was completed he was started on heparin. He was closely monitored in the MICU without further cardiopulmonary arrest or any hemodynamic instability. His alevolar:arterial gradient quickly improved and oxygen support was weaned. He was extubated the second hospital day and transferred out of the MICU on enoxaparin and warfarin. 
Which of the following is true about the role of inferior vena cava (IVC) filters in the setting of massive PE?

1. An IVC filter is indicated in select patients with massive PE if it is felt the next PE could be fatal and an IVC filter additional protection.

2. An IVC filter is recommended for all patients with massive PEs treated with tPA.

3. IVC filter should never be used once PE has already happened

4. There is no indication for IVC filter in the absence of a contra-indication to ongoing anticoagulation such as active bleeding. 


\section{Correct! \\ 4. There is no indication for IVC filter in the absence of a contra-indication to ongoing anticoagulation such as active bleeding.}

The Antithrombotic Therapy and Prevention of Thrombosis Evidence Based Practice Guidelines recommend against the use of IVC filters for patients treated with anticoagulants (2). The Guidelines do recommend the use of IVC filters for patients with a contraindication to anticoagulation. Additionally, the guidelines recommend a conventional course of anticoagulation for patient with an initial contraindication to anticoagulation treated with IVC filters once that contraindication is resolved.

Which of the following is true regarding the role of screening for a hypercoagulable state following a venous thromboembolic event (VTE)?

1. Absolutely essential as the presence of a heritable hypercoagulable state would likely change the recommend time frame for anti-coagulant therapy.

2. More important than screening for a hypercoaguable state is evaluating whether the VTE event was provoked or unprovoked.

3. Screening for a hypercoagulable state has no role in evaluating or managing patients with VTE disease including DVT and/or PE.

4. Screening is only recommended for patients with arterial not venous thromboembolic disease. 


\section{Correct! \\ 2. More important than screening for a hypercoaguable state is evaluating whether the VTE event was provoked or unprovoked.}

In a review of the topic published in 2010, Dalen (4) points out than with the exception of anti-phospholipid antibody syndrome the majority of thrombophilic states including factor $\mathrm{V}$ Leiden and the prothrombin gene mutation do not significantly raise the risk of a second VTE event. Dalen points out that routine thrombophilic work-up following an initial VTE event is unlikely to confer a clinical benefit. An idiopathic or unprovoked VTE event is more important than the heritable states assigning risk. Baglin and colleagues (5) found that the two-year rate of recurrence of an idiopathic VTE event was 19.4\%. For additional risk stratification, a post event d-dimer may be more important in risk stratification. Palareti and colleagues (6) found that after completing three months of conventional anticoagulation, patients with a persistently abnormal D-dimer had a one-year recurrence rate of $10 \%$.

\section{References}

1. Tatco VR, Piedad $\mathrm{HH}$. The validity of hyperdense lumen sign in non-contrast chest CT scans in the detection of pulmonary thromboembolism. Int J Cardiovasc Imaging. 2011;27(3):433-40. [CrossRef] [PubMed]

2. Kearon C, Akl EA, Comerota AJ, et al. Antithrombotic therapy for VTE disease: Antithrombotic Therapy and Prevention of Thrombosis, 9th ed: American College of Chest Physicians Evidence-Based Clinical Practice Guidelines. Chest. 2012;141(2 Suppl):e419S-94S. [CrossRef] [PubMed]

3. Meyer G, Vicaut E, Danays T, et al. Fibrinolysis for patients with intermediaterisk pulmonary embolism. N Engl J Med. 2014;370(15):1402-11. [CrossRef] [PubMed]

4. Dalen JE. Should patients with venous thromboembolism be screened for thrombophilia? Am J Med. 2008;121(6):458-63. [CrossRef] [PubMed]

5. Baglin T, Luddington R, Brown K, Baglin C. Incidence of recurrent venous thromboembolism in relation to clinical and thrombophilic risk factors: prospective cohort study. Lancet. 2003;362(9383):523-6. [CrossRef] [PubMed]

6. Palareti G, Cosmi B, Legnani C, et al. D-dimer testing to determine the duration of anticoagulation therapy. N Engl J Med. 2006;355(17):1780-9. [CrossRef] [PubMed] 